\title{
Correlation between erythropoietin serum levels and erythrocyte susceptibility to lipid peroxidation in elderly with type 2 diabetes
}

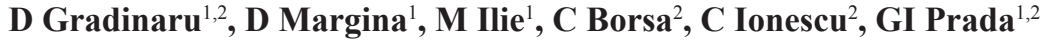 \\ ${ }^{1}$ Department of Biochemistry, Faculty of Pharmacy, Carol Davila-University of Medicine and Pharmacy, \\ Bucharest, Romania \\ ${ }^{2}$ Ana Aslan-National Institute of Gerontology and Geriatrics, Bucharest, Romania
}

Received: September 7, 2014

Accepted: July 15, 2015

\begin{abstract}
Erythropoietin (EPO), a key hormone involved in red blood cell formation has been recently acknowledged for its pleiotropic actions and protective role in ageing and various pathological conditions concurrent with oxidative stress, vascular diseases and metabolic abnormalities such as diabetes mellitus. The aim of the study was to evaluate the relationship between circulating erythropoietin levels and oxidative stress biomarkers, in elderly with type 2 diabetes (T2DM). The study was carried out in 67 subjects with T2DM ( $69 \pm 5$ years; $n=37)$ without anemia, and aged-matched controls $(70 \pm 6$ years; $n=30)$. EPO serum levels, erythrocyte susceptibility to lipid peroxidation (ESP) and total antioxidant capacity (TAC) were evaluated. Lower EPO levels $(p<0.01)$ and higher ESP values $(p$ $<0.001)$ were found in T2DM group, compared to healthy subjects. EPO levels showed significant negative associations with ESP, both in T2DM subjects $(r=-0.565 ; p<0.001)$ and in all study population $(r=-0,600 ; p<$ $0,001 ; n=67)$. In conclusion, we provide new data regarding the cytoprotective effect of EPO exerted at systemic level on erythrocyte membrane, in the particular state of impaired glucose metabolism associated with oxidative stress, in the elderly.
\end{abstract}

Keywords: erythropoietin, type 2 diabetes, oxidative stress, ageing, lipid peroxidation

Erythropoietin (EPO) is a key hormone involved in red blood cell formation in mammalian organisms. Of late years it has been widely acknowledged that erythropoietin's action is not limited to hemopoietic system, as erythropoietin involvement is being extensively evaluated in various pathological conditions concurrent with oxidative stress, vascular diseases, metabolic abnormalities or immune system dysfunction (19). Recent data of biomedical literature has shown that erythropoietin's pleiotropic actions are involved in normal and pathological ageing $(18,20)$.

The Baltimore Longitudinal Study of Aging (BLSA) and the Leiden 85-plus study, have evidenced that EPO serum levels tend to increase with age, probably as physiological response required to maintain adequate red blood cell production (10). In elderly subjects with diabetes mellitus or hypertension, this compensatory mechanism eventually becomes inadequate, leading to a greater risk of developing anemia (12). Additional clinical studies pointed out low or inappropriately normal levels of plasma erythropoietin in diabetic patients with or without anemia $(26,31)$. These abnormalities in erythropoietin biosynthesis in patients with anemia and diabetes mellitus, represent an "inappropriate" response to decreasing

Corresponding author: Denisa Margina, $\mathrm{PhD}$

Department of Biochemistry, Faculty of Pharmacy, Carol Davila-University of Medicine and Pharmacy

6 Taian Vuia street, sector 2, 020956, Bucharest, Romania

Phone: (+40) 74433 9630; Fax: (+40) 21311 1152; E-mail: denisa.margina@umf.ro 
hemoglobin levels (32). Diabetic patients with anemia and decreased EPO levels may be at increased risk of adverse outcomes from diabetic retinopathy, nephropathy, neuropathy, and cardiovascular disease $(24,28)$.

Hyperglycemia, even during short time intervals can lead to increased reactive oxygen species (ROS) generation in endothelial, hepatic, beta-pancreatic cells and in erythrocytes (35). Peroxidation of erythrocyte membrane lipids, exposed to increased ROS impairs their physiological function and reduces red blood cell life-span (17).

Taking into account that besides the ability of stimulating erythrocyte production, erythropoietin has been extensively implicated in normal and pathological cell metabolism regulation through its multiple actions, and that oxidative stress induces dysfunction and ageing of erythrocytes, the aim of this study was to evaluate the relationship between serum erythropoietin and oxidative stress induced erythrocyte vulnerability in elderly subjects with type 2 diabetes.

\section{Materials and Methods}

\section{Subjects}

The study was carried out in 67 patients (34 men and 33 women) aged between 64 and 76 years old, selected by clinical and biochemical criteria from patients hospitalized at Ana Aslan - National Institute of Gerontology and Geriatrics.

Two groups of study patients were selected:

1. Control group which enrolled 30 apparently healthy individuals (18 men and 12 women, with the mean age of $69 \pm 5$ years).

2. Group with type 2 diabetes mellitus (T2DM) according to clinical and biochemical criteria of the American Diabetes Association, which included 37 patients (16 men and 21 women, with the mean age of $70 \pm 6$ years), with nonfasting serum glucose levels $>126 \mathrm{mg}$ / $\mathrm{dL}$, with or without oral antidiabetic medication (3). In the study T2DM subjects were enrolled with good or moderate glycemic control (glycated hemoglobin $[\mathrm{HbA} 1 \mathrm{c}]<8.3 \%$ ), treated with metformin and/or sulfonylurea.

Exclusion criteria were: insulin treatment, prior history of myocardial infarction, stroke, as well as anemia, renal impairment, infections, neoplasic diseases, autoimmune disorders, rheumatoid arthritis, which might determine deficiencies in erythropoietin biosynthesis. We also excluded patients using iron treatment, any hormone replacement therapy, lipid-lowering therapy or antioxidant vitamin supplements.

The study was reviewed and approved by the Ethics Committee of the Ana Aslan National Institute of Gerontology and Geriatrics (approval number 13148/13.01.2014). All study participants provided written informed consent prior to participation in the study.

Anthropometric and clinical characteristics were obtained from each subject after a complete clinical examination. Venous blood samples were collected after an overnight $12 \mathrm{~h}$ fasting period. Whole blood (EDTA as an anticoagulant) was used for erythrocytes isolation and ESP evaluation. The serum samples were obtained after blood centrifugation at 3500 $\mathrm{rpm}$, for $15 \mathrm{~min}$ at $4{ }^{\circ} \mathrm{C}$, according to standard procedures. Serum aliquots were immediately stored at $-70{ }^{\circ} \mathrm{C}$ until analysis for erythropoietin (EPO) and at $-20^{\circ} \mathrm{C}$ for total antioxidant status capacity (TAC) measurements. The biochemical and hematological parameters were determined within the same day. 
Biochemical and hematological parameters

Fasting glucose, total cholesterol, low-density lipoprotein cholesterol (LDL-C), high-density lipoprotein cholesterol (HDL-C), triglycerides (TG), uric acid, creatinine, iron, hemoglobin and hematocrit levels were quantified by standard methods on Konelab 20 (Finland) and Celltac Hematology Analyser (Nihoh, Japan). HbA1c was measured using an ion-exchange HPLC (Biorad Variant II).

Erythropoietin (EPO, normal range 3.22-31.9 $\mathrm{mIU} / \mathrm{mL}$ serum) was measured using a commercially available ELISA kit (EIA-3646, DRG, USA) with intra- and inter-assay coefficients of variation (CV) of 4.8 and $5.1 \%$, respectively.

Erythrocyte susceptibility to lipid peroxidation (ESP) was determined by malonyldialdehyde (MDA) formation in presence of hydrogen peroxide $\left(\mathrm{H}_{2} \mathrm{O}_{2}\right)$ according to the method described by Stocks and Dormandy (29). The extent of ESP was measured by the thiobarbituric acid reactivity assay (TBARS) and expressed as MDA equivalent content, in mmol MDA/g Hb, using a calibration curve with 1,1,3,3-tetramethoxypropane.

Total antioxidant capacity (TAC) was determined using a commercially available kit (ImAnOx, KC 5200 Immundiagnostik AG, Germany). Antioxidants present in the serum sample react with a defined amount of exogenously provided hydrogen peroxide $\left(\mathrm{H}_{2} \mathrm{O}_{2}\right)$. The residual $\mathrm{H}_{2} \mathrm{O}_{2}$ is determined by a colorimetric enzymatic method. Results are expressed as hydrogen-peroxide equivalents $(\mu \mathrm{mol} / \mathrm{L})$ as unit for the antioxidant capacity.

The biochemical and immunoenzymatic tests were performed on a ChemWell 2190 Analyser (Awareness Technology, USA) and Lambda Bio10 Perkin-Elmer Spectrophotometer.

\section{Statistical analysis}

Results were expressed as means + standard deviation (SD). Subsequent to analysis of variance, the control and type 2 diabetes groups (T2DM) were compared by use of two-tail Student's unpaired $t$-test. Differences between groups were considered statistically significant at values of $p<0.05$. The association of serum EPO and ESP levels was analyzed by Pearson's (r) partial correlations using SPSS 7.5 software. The $p$ value of $<0.05$ was considered statistically significant.

\section{Results}

The study was carried out in 67 elderly subjects (49\% women and 51\% men) with T2DM (69 \pm 5 years; $n=37)$ without anemia, and aged-matched controls ( $70 \pm 6$ years; $n=30)$. There was no significant difference in the age or sex distribution between the study groups.

Anthropometric, clinical, hematological and biochemical parameters assessed in the two groups of elderly study-patients were shown in Table I.

Hemoglobin and hematocrit levels were slightly lower (although in the normal range), whereas the values of fasting glucose, total cholesterol, LDL-cholesterol and triglycerides were significantly higher in the group of type 2 diabetes mellitus (T2DM) compared with the control group. Minor differences were also observed for creatinine, uric acid and serum iron levels.

The oxidative stress status was evaluated at systemic level through assessments of erythrocytes susceptibility to lipid peroxidation (ESP) and serum total antioxidant capacity (TAC). The ESP was significantly higher $(p<0.001)$ in T2DM versus control subjects. Also, significantly lower TAC values were obtained in the T2DM group compared to the control (Table II). 
Table I. Clinical, hematological and biochemical characteristics in control and type 2 diabetes mellitus (T2DM) elderly subjects

\begin{tabular}{|c|c|c|c|}
\hline \multirow[t]{2}{*}{ Variable } & $\begin{array}{c}\text { Control group } \\
(n=30)\end{array}$ & $\begin{array}{c}\text { T2DM group } \\
(n=37)\end{array}$ & \multirow[t]{2}{*}{$p$-value } \\
\hline & mean \pm SD & mean \pm SD & \\
\hline Age (years) & $69 \pm 5$ & $70 \pm 6$ & NS \\
\hline Sex (male/female) & $18 / 12$ & $16 / 21$ & \\
\hline Blood pressure (mmHg) & $129 / 80 \pm 11 / 8$ & $147 / 86 \pm 21 / 15$ & NS \\
\hline $\operatorname{BMI}\left(\mathrm{kg} / \mathrm{m}^{2}\right)$ & $23.2 \pm 1.5$ & $24.3 \pm 3.2$ & NS \\
\hline Hemoglobin $(\mathrm{g} / \mathrm{dL})$ & $14.6 \pm 0.8$ & $14.1 \pm 1.1$ & $<0.05$ \\
\hline Hematocrit (\%) & $42 \pm 2$ & $40 \pm 5$ & $<0.05$ \\
\hline Fasting glucose (mg/dL) & $89 \pm 3$ & $142 \pm 17$ & $<0.001$ \\
\hline $\mathrm{HbA1c}(\%)$ & $5.5 \pm 0.3$ & $7.4 \pm 0.8$ & $<0.001$ \\
\hline Total cholesterol (mg/dL) & $198 \pm 19$ & $221 \pm 37$ & $<0.01$ \\
\hline Triglycerides (mg/dL) & $101 \pm 30$ & $156 \pm 57$ & $<0.001$ \\
\hline LDL-C (mg/dL) & $118 \pm 24$ & $143 \pm 15$ & $<0.001$ \\
\hline HDL-C (mg/dL) & $59 \pm 11$ & $50 \pm 15$ & $<0.01$ \\
\hline Iron $(\mu \mathrm{g} / \mathrm{dL})$ & $75 \pm 13$ & $72 \pm 17$ & NS \\
\hline Uric acid $(\mathrm{mg} / \mathrm{dL})$ & $3.32 \pm 1.8$ & $4.42 \pm 2.1$ & $<0.05$ \\
\hline Creatinine (mg/dL) & $0.92 \pm 0.1$ & $1.17 \pm 0.7$ & $<0.05$ \\
\hline
\end{tabular}

$\mathrm{SD}=$ standard deviation; $p$ values represent statistical significance when comparing against the Control group; $\mathrm{NS}=$ non-significant; BMI = Body Mass Index; HbA1c = glycated hemoglobin; LDL-C = low-density lipoprotein cholesterol; HDL-C = high-density lipoprotein cholesterol

Table II. Serum erythropoietin (EPO) levels, erythrocyte susceptibility to lipid peroxidation (ESP) and serum total antioxidant capacity (TAC) in control and type 2 diabetes mellitus (T2DM) elderly subjects

\begin{tabular}{|l|c|c|c|}
\hline \multirow{2}{*}{ Variable } & $\begin{array}{c}\text { Control group } \\
(\boldsymbol{n}=\mathbf{3 0})\end{array}$ & $\begin{array}{c}\text { T2DM group } \\
(\boldsymbol{n}=\mathbf{3 7})\end{array}$ & \multirow{2}{*}{$\boldsymbol{p}$-value } \\
\cline { 2 - 3 } & mean $\pm \mathbf{S D}$ & mean $\pm \mathbf{S D}$ & $<0.01$ \\
\hline $\mathrm{EPO}(\mathrm{ng} / \mathrm{mL})$ & $22.6 \pm 6.8$ & $17.3 \pm 5.8$ & $<0.001$ \\
\hline $\mathrm{ESP}(\mathrm{mmole} / \mathrm{g} \mathrm{Hb})$ & $362 \pm 55$ & $537 \pm 140$ & 0.01 \\
\hline $\mathrm{TAC}(\mu \mathrm{mol} / \mathrm{L})$ & $223 \pm 142$ & $331 \pm 162$ & $<$ \\
\hline
\end{tabular}

$\mathrm{SD}=$ standard deviation; $p$ values represent statistical significance when comparing against the Control group

In elderly subjects with type T2DM, significantly lower serum erythropoietin levels were found compared with the healthy group $(p<0.01)$ (Table II).

The study of interrelations between circulating serum erythropoietin concentrations and erythrocyte susceptibility to peroxidation showed significant negative associations both in T2DM subjects $(r=-0.565 ; p<0.001 ; n=37)$ and all study population $(r=-0,600 ; p<0,001$; $n=67$ ) (Figs 1 and 2). A significant negative correlation was observed even in the control group $(r=-0.590 ; p<0.001 ; n=30)$ (data not shown). 


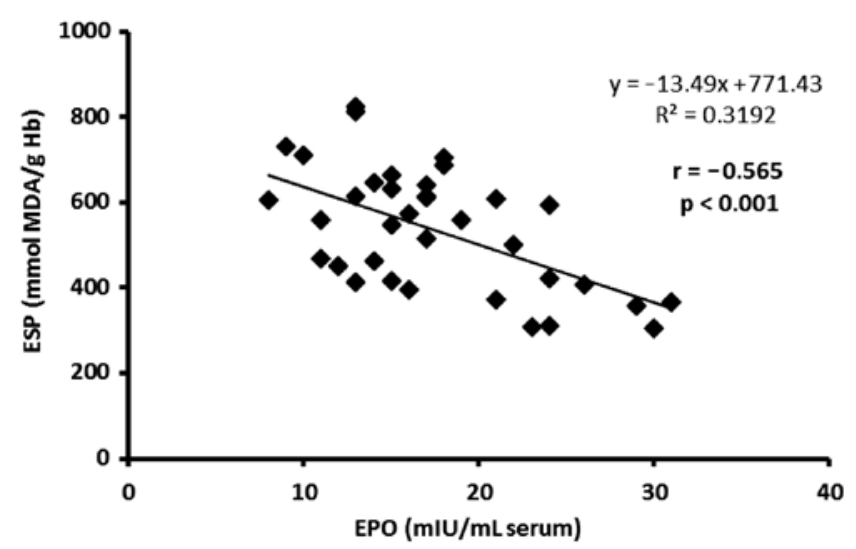

Fig. 1. Correlation between serum erythropoietin (EPO) levels and erythrocyte susceptibility to lipid peroxidation (ESP) in type 2 diabetes mellitus elderly subjects $(n=37)$

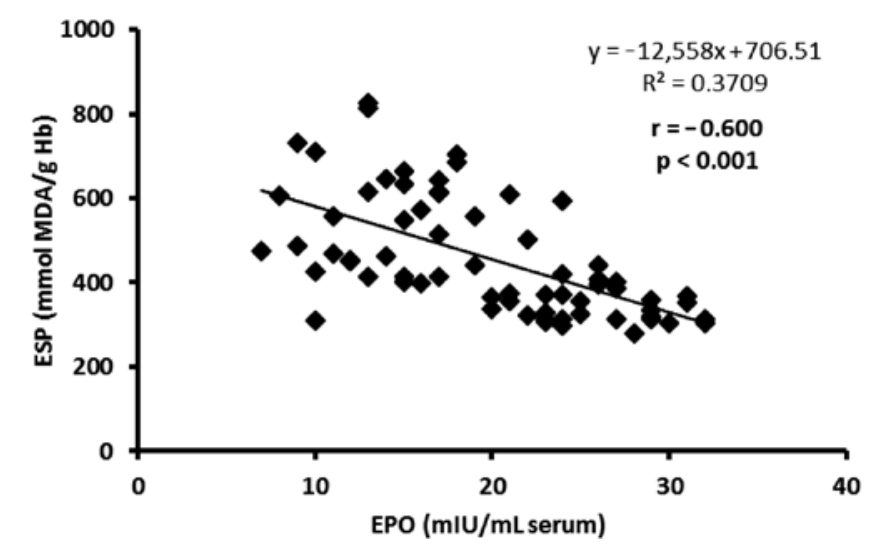

Fig. 2. Correlation between serum erythropoietin (EPO) levels and erythrocyte susceptibility to lipid peroxidation (ESP) for all study subjects $(n=67)$

\section{Discussion}

Erythropoietin is among the first hormonal factors discovered in humans, involved in both erythropoiesis and various cytoprotective pathways. In basic experimental research and clinical studies, erythropoietin has been strongly associated with modulation of cellular metabolism $(19,20)$. Diabetes mellitus, a major public health problem, comes also as main evidence when cellular dysfunction related to cell metabolism is under scrutiny (21). Furthermore, metabolic disturbances and oxidative stress appear to be strongly related in the etiopathogeny of type 2 diabetes.

Several studies evidenced that oxidative status was improved by EPO treatment of anemia $(2,11,25)$. EPO therapy may exert its antioxidant effect, by modulating the action of erythrocyte antioxidant enzymes such as superoxide dismutase and glutathione 
peroxidase (25). Also, recent results showed that EPO treatment increased the GSH levels in both erythrocytes and platelets, in $\beta$-thalassemic patients (2). However, the relationships between EPO and erythrocyte oxidative stress in elderly subjects with diabetes have been less explored.

Normal erythrocyte function depends on integrity of the erythrocyte membrane, which is a target for numerous toxic prooxidant factors, including chronic hyperglycemia $(22,30)$. Erythrocyte membrane is among the main targets for oxygen radicals or peroxides as it contains both polyunsaturated fatty acids (PUFA), which are the most susceptible substrate for lipid peroxidation as well as prooxidant catalysts such as $\mathrm{Fe}^{2+}$ and hemoproteins $(23,33)$. Hence, the erythrocyte susceptibility to peroxidation represents an informative biomarker on red blood cell mechanisms and capability of antioxidant protection against free radicals.

Therefore, in this study we aimed to investigate the correlation between circulating EPO levels and systemic oxidative stress biomarkers, in elderly with type 2 diabetes (T2DM). In view of this purpose, we evaluated the erythrocytes susceptibility to lipid peroxidation (ESP) and serum total antioxidant capacity (TAC).

Results showed that elderly T2DM subjects had a decreased antioxidant defense, in both serum samples and erythrocytes, compared to healthy subjects, which is in accordance with previous studies $(14,15)$. Also, lower levels of EPO were found in subjects with impaired glucose metabolism, compared with age-matched controls. The main finding of our study showed that erythropoietin levels correlated inversely and significantly with oxidative stress exerted at erythrocyte level (ESP), even though hemoglobin, the circulating erythrocyte mass and serum iron levels were comparable. Also, lower levels of EPO in T2DM group were associated with a significant decrease in serum antioxidant capacity (TAC). Subsequently to establishing these associations, the following questions could be raised: to what extent diabetes determine a decrease in EPO biosynthesis and to what extent diabetes per se can initiate oxidative damage of erythrocyte membrane? And not in the least, which might be the mechanisms of the antioxidant EPO protection expressed at erythrocyte level?

Diabetes-related chronic hyperglycemia can lead to a hypoxic environment in the renal interstitium, which results in impaired production of erythropoietin by the peritubular fibroblasts and in diabetes nephropathy, subsequent anemia (28). The distinction between T2DM patients with anemia and those with diabetic nephropathy in whom anemia has not been evidenced, lies in a defect of "anemia-sensing" mechanisms particular to these later mentioned patients, and that may contribute to EPO deficiency and explain low EPO levels (9). In ageing, the decline in the capacity to produce EPO could be due to a mechanism distinct from impaired renal excretory function. Certain ageing-associated disorders such as diabetes mellitus and hypertension, could accentuate the impairment in EPO secretion (12).

Due to their physiological roles, erythrocytes are continuously exposed to oxidative stress. Results obtained confirm observations of literature regarding the damaging effects of hyperglycemia mediated or stimulated by oxygen species. Mechanisms could be the following: 1) glucose oxidation pathways generate oxygen free radicals; 2) hyperglycemia deteriorates antioxidant capacity, and 3) mitochondria-derived reactive oxygen species is generated in hyperglycemic states $(6,19,21)$.

Moreover, in kidney cells hyperglycemia increases mitochondrial ROS production which alters the transcription factors involved in the control of hypoxia and oxidative stress response in relation with the renal hypoxia-inducible factor (HIF) pathway, involved in erythropoiesis (19). Research has extended to the effects of EPO in regulating oxidative 
metabolism and mitochondrial function, the interactions between EPO and important energy regulation factors, and the protective role of EPO from stresses that are related to metabolism, which have been recently reviewed $(34,35)$.

EPO may exert its antioxidative effects directly by involvement in intracellular mechanisms such as up-regulation of hem oxygenase-1 expression, superoxide dismutase, catalase and glutathione peroxidase activities, and by inhibiting reactive oxygen species generation. Recent studies found the recombinant form of EPO (rHuEPO) to be a potent scavenger of $\mathrm{HO}^{*}$ with the capacity to inhibit Fenton chemistry through catalytic iron chelation. Its ability to scavenge peroxyl (ROO') radicals, evaluated in vitro, was also superior compared to other antioxidants (5). The indirect antioxidant EPO action could be achieved through iron depletion and thus limitation of iron-dependent oxidative injury, and by increasing new red blood cells (RBC) loaded with a significant amount of antioxidants $(1,2,11,16)$.

Previous studies pointed out also an inverse association between hemoglobin concentrations and erythrocyte membrane MDA (13). In our study population hemoglobin and serum iron concentrations were comparable, and within normal ranges, therefore we could suggest that mainly erythropoietin and not hemoglobin, nor iron determined the changes in oxidative stress parameters.

Recent in vitro and in vivo studies underline the antioxidative EPO effect in various cell systems: erythrocytes, neurons, microglia, astrocytes, cerebral endothelial cells, cardiomyocytes, retinal cell $(2,11,16,20)$. In in vitro studies on blood vessel cells exposed to increased glucose concentrations a strong cyto-protective EPO effect was noticed (8). Similarly to insulin, also for EPO protective effects were demonstrated, subsequent to its administration to diabetic and non-diabetic patients, with resistant, severe congestive heart failure (27). EPO administration can significantly improve survival of endothelial cells and moreover, EPO can block DNA degradation in the course of apoptosis of endothelial cells under hyperglycemia conditions, similarly to other experimental models of oxidative stress in cultured cardiac and endothelial cells (4). A recent study which investigated the timedependent effects of EPO therapy on oxidative stress parameters of hemodialysis patients showed that long term administration of EPO attenuated the lipid peroxidation process and restored the levels of antioxidants (11). New in vivo research showed potent protective effects of EPO against diabetes development by direct effects of EPO on $\beta$-cells by increasing $\beta$-cell mass through anti-apoptotic, proliferative and angiogenic mechanisms (7).

The strong inverse association between EPO levels and ESP pointed out in our study, in T2DM, could reflect the diminished RBC membrane cytoprotection by reducing the strength of total antioxidant potential at systemic level. Further in vitro experimental models and in vivo studies are needed to demonstrate the precise mechanisms.

The limitations of this study could be the small population sample and the global assessment of oxidative stress damage exerted on erythrocytes (ESP) without specific evaluations of biomarkers of erythrocyte antioxidant defense, such as superoxide dismutase, glutathione, glutathione peroxidase and catalase. As oxidative stress in diabetes is suggested to be associated also with chronic low-grade inflammation, another limitation of the study is the lack in the assessment of sensitive inflammatory markers (such as hsCRP, IL-6, TNF- $\alpha$ ), therefore we could not point out relationships between decreased EPO serum levels, oxidative stress markers and inflammation. 
The present study provides new data regarding the protective effect of EPO expressed at systemic level, and the relationships between circulating EPO levels, impaired glucose metabolism and erythrocyte susceptibility to oxidative damage. Since administration of antioxidants in the course of hyperglycemia can block free radical generation and reduce oxidative stress, EPO could offer an alternative therapy appealing in view of maintenance of normal cell metabolism and appropriate membrane potential. Hence, inhibition of oxidative damage of cell components could represent a new modality of EPO therapeutic intervention in diabetes. In this sense, in the future both glucose lowering therapy as well as judicious early supplementation with erythropoietin could lead to delay or block the development of anemia and vascular complications in elderly diabetic patients.

\section{Acknowledgements}

The authors are grateful for the opportunity to make use of the equipment obtained under the framework of the EUFP7 Project "MARK-AGE, European Study to Establish Biomarkers of Human Ageing”.

\section{REFERENCES}

1. Akbulut S, Gurbilek M, Kryici A, Akoz M, Alintepe L, Karakuscu A, Topcu C: The effect of erythropoietin application on erythrocyte Na,K-ATPase activities in patients with diabetic polyneuropathy. Turk. J. Biochem. 38, 13-17 (2013)

2. Amer J, Dana M, Fibach E: The antioxidant effect of erythropoietin on thalassemic blood cells. Anemia 2010, ID 978710 (2010)

3. American Diabetes Association: Diagnosis and classification of diabetes mellitus. Diabetes Care 34, 562-569 (2011)

4. Avasarala JR, Konduru SS: Recombinant erythropoietin down-regulates IL-6 and CXCR4 genes in TNF-alphatreated primary cultures of human microvascular endothelial cells: implications for multiple sclerosis. J. Mol. Neurosci. 25, 183-189 (2005)

5. Bailey DM, Lundby C, Berg RM, Taudorf S, Rahmouni H, Gutowski M, Mulholland CW, Sullivan JL, Swenson ER, McEneny J, Young IS, Pedersen BK, Møller K, Pietri S, Culcasi M: On the antioxidant properties of erythropoietin and its association with the oxidative-nitrosative stress response to hypoxia in humans. Acta Physiol. (Oxf). 212, 175-187 (2014)

6. Bonnefont-Rousselot D, Bastard JP, Jaudon MC, Delattre J: Consequences of the diabetic status on the oxidant/ antioxidant balance. Diabetes Metab. 26, 163-176 (2000)

7. Choi D, Schroer SA, Lu SY, Wang L, Wu X, Liu Y, Zhang Y, Gaisano HY, Wagner KU, Wu H, Retnakaran R, Woo M: Erythropoietin protects against diabetes through direct effects on pancreatic $\beta$ cells. J. Exp. Med. 207, 2831-2842 (2010)

8. Chong ZZ, Shang YC, Maiese K: Vascular injury during elevated glucose can be mitigated by erythropoietin and Wnt signaling. Curr. Neurovasc. Res. 4, 194-204 (2007)

9. Craig KJ, Williams JD, Riley SG, Smith H, Owens DR, Worthing D, Cavill I, Phillips AO: Anemia and diabetes in the absence of nephropathy. Diabetes Care 28, 1118-1123 (2005)

10. den Elzen WP, Willems JM, Westendorp RG, de Craen AJ, Blauw GJ, Ferrucci L, Assendelft WJ, Gussekloo J: Effect of erythropoietin levels on mortality in old age, the Leiden 85-plus Study. CMAJ 182, 1953-1958 (2010)

11. Dimitrijevic ZM, Cvetkovic TP, Djordjevic VM, Pavlovic DD, Stefanovic NZ, Stojanovic IR, Paunovic GJ, Velickovic-Radovanovic RM: How the duration period of erythropoietin treatment influences the oxidative status of hemodialysis patients. Int. J. Med. Sci. 9, 808-815 (2012)

12. Ershler WB, Sheng S, McKelvey J, Artz AS, Denduluri N, Tecson J, Taub DD, Brant LJ, Ferrucci L, Longo DL: Serum erythropoietin and aging: a longitudinal analysis. J. Am. Geriatr. Soc. 53, 1360-1365 (2005)

13. Gallucci MT, Lubrano R, Meloni C, Morosetti M, Manca di Villahermosa S, Scoppi P, Palombo G, Castello MA, Casciani CU: Red blood cell membrane lipid peroxidation and resistance to erythropoietin therapy in hemodialysis patients. Clin. Nephrol. 52, 239-245 (1999) 
14. Gradinaru D, Borsa C, Ionescu C, Margina D, Prada GI, Jansen E: Vitamin D status and oxidative stress markers in the elderly with impaired fasting glucose and type 2 diabetes mellitus. Aging Clin. Exp. Res. 24, 595-602 (2012)

15. Gradinaru D, Borsa C, Ionescu C, Margina D: Advanced oxidative and glycoxidative protein damage markers in the elderly with type 2 diabetes. J. Proteomics 92, 313-322 (2013)

16. Katavetin P, Tungsanga K, Eiam-Ong S, Nagaku M: Antioxidative effects of erythropoietin. Kidney Int. 72 , $10-15$ (2007)

17. Lang F, Lang KS, Lang PA, Huber SM, Wieder T: Mechanisms and significance of eryptosis. Antioxid. Redox Signal. 8, 1183-1192 (2006)

18. Lombardero M, Kovacs K, Scheithauer BW: Erythropoietin: a hormone with multiple functions. Pathobiology 78, 41-53 (2011)

19. Maiese K, Chong ZZ, Hou J, Shang YC: Erythropoietin and oxidative stress. Curr. Neurovasc. Res. 5, 125-142 (2008)

20. Maiese K, Chong ZZ, Li F, Shang YC: Erythropoietin: elucidating new cellular targets that broaden therapeutic strategies. Prog. Neurobiol. 85, 194-213 (2008)

21. Maiese K, Chong ZZ, Shang YC: Mechanistic insights into diabetes mellitus and oxidative stress. Curr. Med. Chem. 14, 1729-1738 (2007)

22. Marotta F, Pavasuthipaisit K, Yoshida C, Albergati F, Marandola P: Relationship between aging and susceptibility of erythrocytes to oxidative damage: in view of nutraceutical interventions. Rejuvenation Res. 9, 227-230 (2006)

23. Martin-Ventura JL, Madrigal-Matute J, Martinez-Pinna R, Ramos-Mozo P, Blanco-Colio LM, Moreno JA, Tarin C, Burillo E, Fernandez-Garcia CE, Egido J, Meilhac O, Michel JB: Erythrocytes, leukocytes and platelets as a source of oxidative stress in chronic vascular diseases: detoxifying mechanisms and potential therapeutic options. Thromb. Haemost. 108, 435-442 (2012)

24. McGill JB, Bell DS: Anemia and the role of erythropoietin in diabetes. J. Diabetes Complications 20, 262-272 (2006)

25. Mimic-Oka J, Simic T, Djukanovic L: Epoetin treatment improves red blood cell and plasma antioxidant capacity in hemodialysis patients. Ren. Fail. 24, 77-87 (2002)

26. Mojiminiyi OA, Abdella NA, Zaki MY, El Gebely SA, Mohamedi HM, Aldhahi WA: Prevalence and associations of low plasma erythropoietin in patients with Type 2 diabetes mellitus. Diabet. Med. 23, 839-844 (2006)

27. Silverberg DS, Wexler D, Iaina A, Schwartz D: The interaction between heart failure and other heart diseases, renal failure, and anemia. Semin. Nephrol. 26, 296-306 (2006)

28. Singh DK, Winocour P, Farrington K: Erythropoietic stress and anemia in diabetes mellitus. Nat. Rev. Endocrinol. 5, 204-210 (2009)

29. Stocks J, Dormandy TL: The autooxidation of human red cell lipids induced by hydrogen peroxide. Br. J. Haematol. 20, 95-111 (1971)

30. Stocks J, Offerman EL, Modell CB, Dormandy TL: The susceptibility to autooxidation of human red cell lipids in health and disease. Br. J. Haematol. 23, 713-724 (1972)

31. Symeonidis A, Kouraklis-Symeonidis A, Psiroyiannis A, Leotsinidis M, Kyriazopoulou V, Vassilakos P, Vagenakis A, Zoumbos N: Inappropriately low erythropoietin response for the degree of anemia in patients with noninsulin-dependent diabetes mellitus. Ann. Hematol. 85, 79-85 (2006)

32. Thomas MC, Cooper ME, Tsalamandris C, MacIsaac R, Jerums G: Anemia with impaired erythropoietin response in diabetic patients. Arch. Intern. Med. 165, 466-469 (2005)

33. Tsantes AE, Bonovas S, Travlou A, Sitaras NM: Redox imbalance, macrocytosis, and RBC homeostasis. Antioxid. Redox Signal. 8, 1205-1216 (2006)

34. Wang L, Di L, Noguchi T: Erythropoietin, a novel versatile player regulating energy metabolism beyond the erythroid system. Int. J. Biol. Sci. 10, 921-939 (2014)

35. Yano M, Hasegawa G, Ishii M, Yamasaki M, Fukui M, Nakamura N, Yoshikawa T: Short-term exposure of high glucose concentration induces generation of reactive oxygen species in endothelial cells: implication for the oxidative stress associated with postprandial hyperglycemia. Redox. Rep. 9, 111-116 (2004) 